\title{
Multi Laser Displacement Sensors Based Three-dimensional Pavement Measuring Technology
}

\author{
Jianfeng Wang ${ }^{1,}$ a, Xueqin $\mathrm{Wu}^{2, \mathrm{~b}}$ and Wenfeng Song ${ }^{2, \mathrm{c}}$ \\ ${ }^{1}$ Shaanxi Road Traffic Intelligent Detection and Equipment Engineering Technology Research \\ Center, Chang'an University, Xi'an 710064, China; \\ ${ }^{2}$ School of Automobile, Chang'an University, Xi'an 710064, China. \\ acsfxzx@163.com, b2812262921@qq.com, c2273562947@qq.com
}

\begin{abstract}
A three-dimensional measuring method for pavement based on multi laser displacement sensors is proposed to establish a complete and powerful database of pavement shape. Model of the measuring system based on this method is established and analyzed. Data generated by sensors, including data of laser displacement sensors, acceleration sensors and gyroscope, is transmitted according to TCP/IP Protocol. The back-end computer fuses the data and establishes the databank of 3-D pavement shape. Experimental results of pavement shape measurement using gradienter and the system based on this method proved that the application and accuracy of this method are satisfied.
\end{abstract}

Keywords: Pavement; Data fusion; Ruts measuring; 3-D module; Laser displacement sensor.

\section{Introduction}

The flatness of pavement affects driving safety, comfort, and the efficiency of transportation [12]. International roughness index (IRI) is commonly adopted as the indicator for pavement measurement, which is given by calculating the cross-section curve of road [3-4]. Depth of ruts, which is worn by vehicles and affects the quality of road and driving security, can be calculated according to data of road cross-sections shape.

IRI and the depth of ruts are important for transportation related organizations. However, a database of 3-D pavement-shape, which gives multi indicators of whole road system, is more attracting. The quality of pavement can be evaluated with this database visibly. The detail information of any longitudinal or cross section of pavement can be given by processing the database. IRI and rut-depth at any given time and position can be calculated.

\section{Theory of measurement}

Shape of pavement in the database is described by an array, in which components are coordinates in $\mathrm{X}-\mathrm{Y}-\mathrm{Z}$ rectangle coordinate system. Where $\mathrm{X}$ axis refers to the longitudinal direction of road, $\mathrm{Y}$ axis refers to the cross direction, $Z$ refers to elevation of pavement. Data of pavement shape is stored in external storage to establish the database of pavement shape [5-6].

A robust beam is adopted in this method as the framework of sensors. Multi laser displacement sensors (LDSs) are mounted on the beam to measure the elevation of pavement. Acceleration sensors are mounted at the ends of the pavement to measure vibration of the beam. Displacement and inclination of the beam can be estimated by processing the vibration signal, which provides information to correct the data acquired by LDSs. Gyroscopes are mounted on the vehicle to measure the geographic trend of road.

Data of the geographic trend measured by gyroscope and elevation of pavement measured by LDS and acceleration sensors in a wavelet-length is fused to calculate the 3-D shape of pavement.

It is necessary that there must be an invariable benchmark for the rigid beam to work. But as is shown in Fig. 1, the rigid beam is inevitable to be affected by vibration of system. The beam will float and rotate around the idea benchmark repeatedly, which undermines the accuracy of measurement.

Set the shape curve of road cross-section as $\mathrm{T}$, which is stated as

$T_{i}=f(y)=\left\{\left(x_{i}, y_{1}, z_{i 1}\right) \times\left(x_{i}, y_{2}, z_{i 2}\right) \cdots\left(x_{i}, y_{m}, z_{i m}\right)\right\}$ 


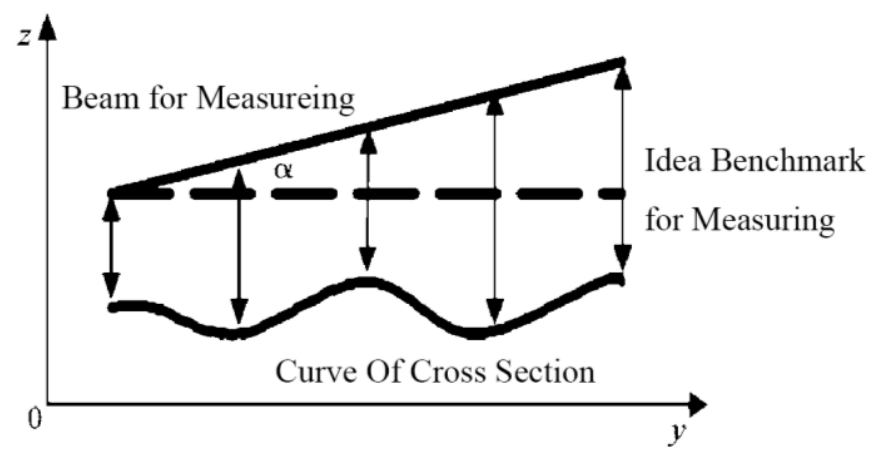

Fig. 1 Beam for measuring inclines

When the beam vibrates, set the curve of the cross-section measuring by system as $T_{i}^{\prime}$ :

$T_{i}^{\prime}=T_{i}+y_{i} \tan \alpha+b_{i}=\left\{\left(x_{i}, y_{1}, z_{i 1}^{\prime}\right) \times\left(x_{i}, y_{2}, z_{i 2}^{\prime}\right) \cdots\left(x_{i}, y_{m}, z_{i m}^{\prime}\right)\right\}$

Where $\alpha$ the inclination of the beam and idea benchmark is, $b$ is the intercept of the beam relative to idea benchmark. $\alpha$ and $b$ are essential to get the information of the cross section.

The linear correction method is usually used to figure out the actual curve of the cross section. $\operatorname{Drop}\left(\mathrm{y}_{\mathrm{i}}, \mathrm{z}_{\mathrm{m}}\right)$ into Eq. (3)

$z_{i j}^{\prime}=y_{j} \tan _{i}+b_{i}$

An equation group is then given by

$$
\left\{\begin{array}{c}
z_{i 1}^{\prime}=y_{1} \tan \alpha_{i}+b_{i} \\
z_{i 2}^{\prime}=y_{2} \tan \alpha_{i}+b_{i} \\
\vdots \\
z_{\text {in }}^{\prime}=y_{n} \tan _{i}+b_{i}
\end{array}\right\}
$$

The least square method is adopted to calculate $\alpha$ and $b$. Put $\alpha$ and $b$ into Eq. (2) and get the corrected curve $T^{\prime \prime}$

$T^{\prime \prime}=T^{\prime}-(y \tan \alpha+b)$

Notice that $\alpha$ and $b$ calculated in Eq. (4) are not given by a common benchmark, but a float benchmark, which indicates Eq. (5) can only give the curve of a single cross-section. To get the 3-D shape of the whole pavement, the elevation of longitudinal-sections of pavement must be given [78].

To get the 3-D shape of pavement, the inclination and elevation change relative to the idea benchmark of the rigid beam must be given. As is shown in Fig. 2, control point 1 and 2 for longitudinal-section are introduced and add elevation of longitudinal-sections of pavement into the model.

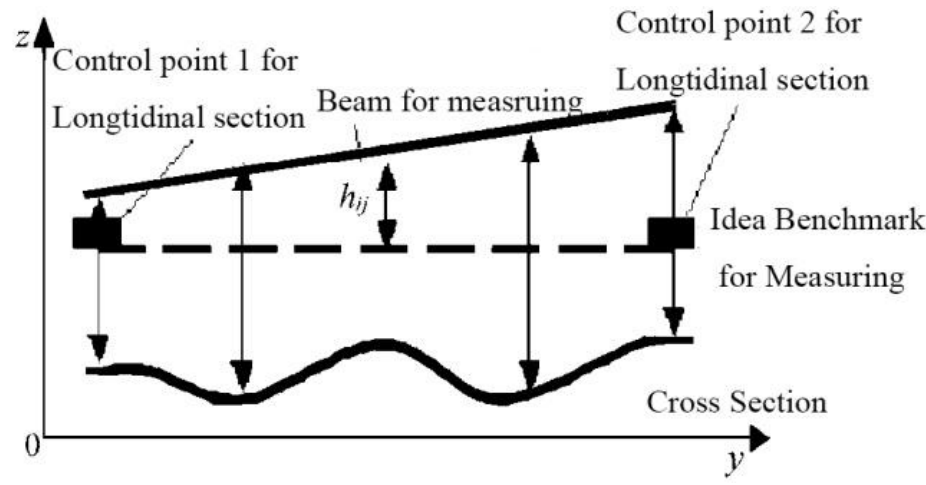

Fig. 2 The new model combining information of cross and longitudinal section.

Set position of point 1 as $\left(x_{i}, y_{i}, z_{i 1}\right)$ and of point 2 as $\left(x_{i}, y_{m}, z_{i m}\right)$, where $i$ refers to the sequence number of cross sections. Inclination $\alpha_{i}$ and elevation $h_{i j}$ relative to the benchmark at every point of the beam can be calculated according to the displacement of the two control points. $\alpha_{i}$ and $h_{i j}$ are given by

$$
\tan \alpha_{i}=\frac{\left(z_{i m}-z_{i m}^{\prime}\right)-\left(z_{i l}-z_{i 1}^{\prime}\right)}{y_{m}-y_{1}}
$$




$$
h_{i j}=\left(z_{i 1}-z_{i 1}^{\prime}\right)+\frac{j-1}{m} \times\left[\left(z_{i m}-z_{i m}^{\prime}\right)-\left(z_{i 1}-z_{i 1}^{\prime}\right)\right]
$$

Where $j$ is the sequence number of points on the beam, $m$ is the total number of points measured by system, $h_{i j}$ is the vibration factor at every point, $z_{i m}$ is the relative elevation of pavement measured by the incline beam.

A derivation of Eq. (2) is given:

$$
T_{i}=T_{i}^{\prime}-h_{i j}=\left\{\left(x_{i}, y_{1}, z_{i 1}^{\prime}-h_{i 1}\right) \times\left(x_{i}, y_{2}, z_{i 2}^{\prime}-h_{i 2}\right) \cdots\left(x_{i}, y_{m}, z_{i m}^{\prime}-h_{i m}^{\prime}\right)\right\}
$$

The 3-D shape rebuilt by system is shown in Fig. 3. The 3-D surface $R$ of pavement is stated as follow

$$
R\left\{\begin{array}{c}
T_{1} \\
T_{2} \\
\vdots \\
T_{n}
\end{array}\right\}=\left\{\begin{array}{c}
\left(x_{i}, y_{1}, z_{11}^{\prime}-h_{11}\right) \times\left(x_{i}, y_{2}, z_{12}^{\prime}-h_{i 2}\right) \cdots\left(x_{i}, y_{m}, z_{1 m}^{\prime}-h_{1 m}^{\prime}\right) \\
\left(x_{2}, y_{1}, z_{21}^{\prime}-h_{21}\right) \times\left(x_{2}, y_{2}, z_{22}^{\prime}-h_{22}\right) \cdots\left(x_{2}, y_{m}, z_{2 m}^{\prime}-h_{2 m}^{\prime}\right) \\
\vdots \\
\left(x_{n}, y_{1}, z_{n 1}^{\prime}-h_{n 1}\right) \times\left(x_{n}, y_{2}, z_{n 2}^{\prime}-h_{n 2}\right) \cdots\left(x_{n}, y_{m}, z_{n m}^{\prime}-h_{n m}^{\prime}\right) \\
\text { curve of } \\
y
\end{array}\right.
$$

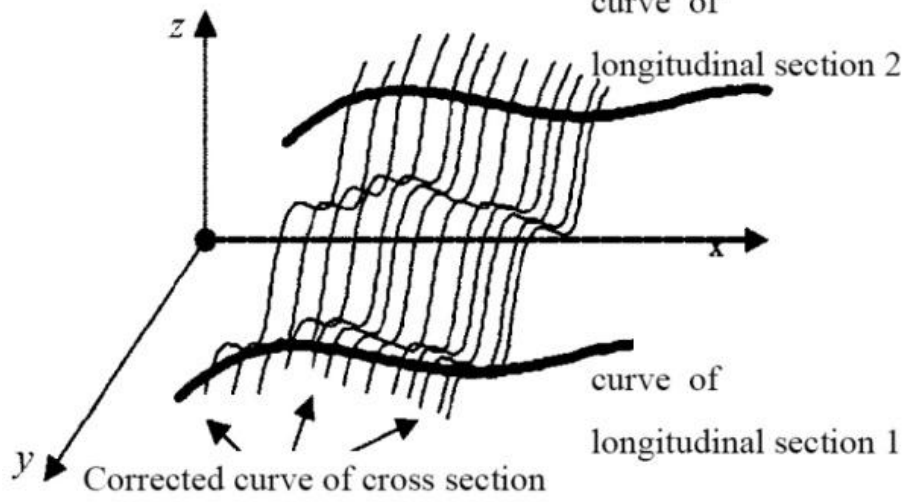

Fig. 3 The 3-D shape of pavement

\section{Measuring System}

Width of an expressway-lane is limited to $3.75 \mathrm{~m}$ in China and theoretically the rigid beam should be 3.75 meter long either. But a beam with such length may threaten other automobiles when the measuring vehicle running on the road. So the rigid beam is designed to have a two-layer construction, as is shown in Fig. 4. A mechanical vibration absorb system is designed to suspend the beam on the vehicle chassis to minimize the impact of vibration. The down-layer of the beam is fixed but up-layer can stretch. The up-layer can draw in when measuring system doesn't work.

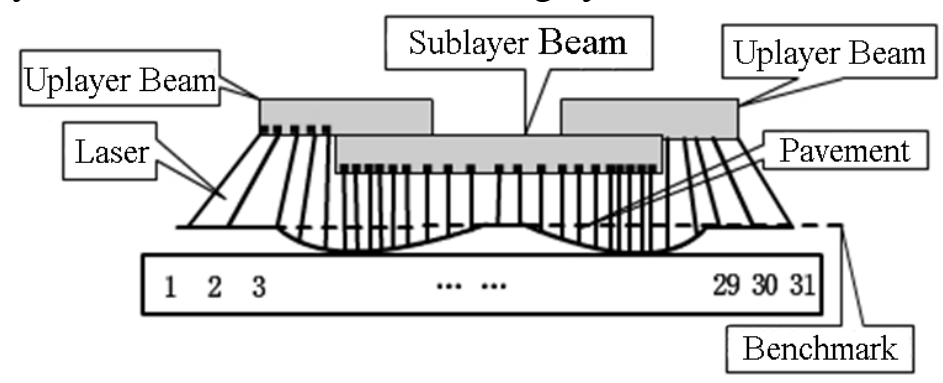

Fig. 4 The structure of beam for measuring

It's important that the LDSs should not be distributed on the beam equidistantly. Spacing among sensors over the ruts is smaller, while spacing close to the ends of beam is larger. LDSs closing to the ends are installed in inclined to give system a 3.75-meter-width scanning range.

\section{Application and Examination}

To test the measuring precision of system, we chose six 30-meter-long and 3.75-meter-wide lanes for experiment. Firstly, we used gradienters to measure the elevation of the pavement along the 
longitudinal direction per 0.25 meter and cross direction per 0.25 meter. The 3 -D pavement measuring system measured the elevation of these lanes later.

The system got data about the longitudinal sections, which is shown in Fig. 5(b). Additionally, the measuring positions of the system were the same with of gradienters. Data measured with gradienters is shown in Fig. 5(a). Processing the module rebuilt by computer, we got the curve of cross section, which is shown in Fig. 5(d). Curve measured with gradienters is shown in Fig. 5(c).
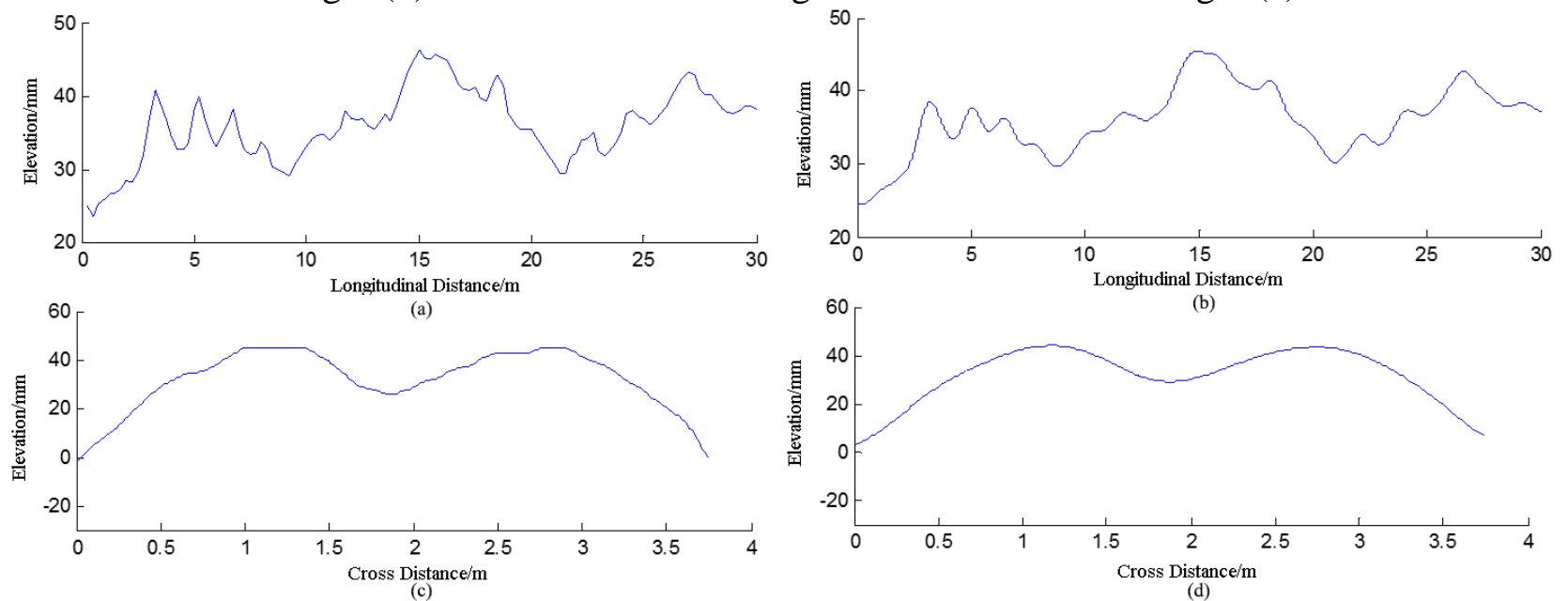

Fig.5 Elevation measured with gradienters and the 3-D pavement measuring system

The system was tested on the expressway from Anyang to Hebi, Weiwu to Gulang and Sanmenxia to Guanyintang. The vehicle measured at a speed about $72 \mathrm{~km} / \mathrm{h}$, with a 3.75 -meter-wide measuring range and $0.05 \mathrm{~m}$ sampling interval. By analyzing the measurement results of different instruments on same roads, we conclude that the relative error of IRI, which relates with longitudinal sections, is smaller than $3 \%$, and the relative error of ruts depth, which relates with cross sections, is smaller than $2 \%$.

\section{Conclusion}

This paper presents a measuring system basing on multi-sensor data fusion for pavement. It transmits data according to TCP/IP protocol. The software can rebuild a precise 3-D module of pavement and get information of the cross or longitudinal sections. The values of IRI and depth of ruts are figured out according the information of these sections and the correlation of different positions of roads is presented visibly by the system, which are then be used to build an integral data bank for departments and organizations.

\section{Acknowledgements}

This work was financially supported by the Funds for Natural Science of Shaanxi Province and the Fundamental Research Funds for the Central Universities in Chang'an University (No.310822163310, No.310822161125, No.310822151122, No.310822151123, No.310822151127, No.310822151124).

\section{References}

[1]. Wei Wang, Shuo Liu, Qizhi Wang, Wei Yuan, Mingzhang Chen, Xiaotian Hao, Shuai Ma, Xuanyu Liang, The Impact of Traffic-Induced Bridge Vibration on Rapid Repairing HighPerformance Concrete for Bridge Deck Pavement Repairs, Advances in Materials Science and Engineering, Vol. 2014, 2014.

[2]. Shuangqing Tang, Zhou Jiang, Qiongqing Bo, Dongwei Zhou, The Application of Virtual Simulation Technology in Studying Vehicle Vibration Characteristics on Random Road, 5th 
International Conference on Information Engineering for mechanics and Materials, pp. 1558$1562,2015$.

[3]. Xiaodan Wei, Research on the International Roughness Index (IRI) as the Pavement Roughness Index, Journal of Highway and Transportation Research and Development, Vol. 16, no. 1, pp. 9$13,1999$.

[4]. Sayers M W, Gullespoe T D, Oparerson WD. Guidelines for Conducting and Calibrating Road Roughness Measurements. World Bank Technical Paper Number 46, The World Bank, pp.6-9, 1986.

[5]. Klas Bogsjöa, Krzysztof Podgórskib, Igor Rychlikc, Models for Road Surface Roughness, Vehicle System Dynamics: International Journal of Vehicle Mechanics and Mobility, vol. 50, no. 5, pp.725-747, 2012.

[6]. Thomas F. Statistical approach to road segmentation, Journal of Transportation Engineering, vol.129, no.3, pp.300-308, 2003.

[7]. Guirong Ma, Study on the Principle and Method of Road Tree-Dimension Measurement System, School of Information Engineering, Chang'an University, 2008.

[8]. Chiu Liu, Herman R. Road profiles, Vehicle Dynamics and Human Judgment of Serviceability of Roads Spectral Frequency Domain Analysis, Journal of Transportation Engineering, vol.33, no.4. Pp.155-161, 1998. 